\title{
A protocol for custom CRISPR Cas9 donor vector construction to truncate genes in mammalian cells using pcDNA3 backbone
}

\author{
Neftali Vazquez ${ }^{1 \dagger}$, Lilia Sanchez ${ }^{1 \dagger}$, Rebecca Marks ${ }^{1 \dagger}$, Eduardo Martinez ${ }^{1}$, Victor Fanniel ${ }^{1}$, Alma Lopez ${ }^{1}$, \\ Andrea Salinas ${ }^{1}$, Itzel Flores', Jesse Hirschmann' ${ }^{1}$, Robert Gilkerson' ${ }^{1}$, Erin Schuenzel ${ }^{1}$, Robert Dearth', \\ Reginald Halaby ${ }^{2}$, Wendy Innis-Whitehouse ${ }^{3}$ and Megan Keniry ${ }^{1^{*}}$ (])
}

\begin{abstract}
Background: Clustered regularly interspaced short palindromic repeat (CRISPR) RNA-guided adaptive immune systems are found in prokaryotes to defend cells from foreign DNA. CRISPR Cas9 systems have been modified and employed as genome editing tools in wide ranging organisms. Here, we provide a detailed protocol to truncate genes in mammalian cells using CRISPR Cas9 editing. We describe custom donor vector construction using Gibson assembly with the commonly utilized pcDNA3 vector as the backbone.
\end{abstract}

Results: We describe a step-by-step method to truncate genes of interest in mammalian cell lines using custommade donor vectors. Our method employs 2 guide RNAs, mutant Cas9D10A nickase (Cas9=CRISPR associated sequence 9), and a custom-made donor vector for homologous recombination to precisely truncate a gene of interest with a selectable neomycin resistance cassette (NPTII: Neomycin Phosphotransferase II). We provide a detailed protocol on how to design and construct a custom donor vector using Gibson assembly (and the commonly utilized pcDNA3 vector as the backbone) allowing researchers to obtain specific gene modifications of interest (gene truncation, gene deletion, epitope tagging or knock-in mutation). Selection of mutants in mammalian cell lines with G418 (Geneticin) combined with several screening methods: western blot analysis, polymerase chain reaction, and Sanger sequencing resulted in streamlined mutant isolation. Proof of principle experiments were done in several mammalian cell lines.

Conclusions: Here we describe a detailed protocol to employ CRISPR Cas 9 genome editing to truncate genes of interest using the commonly employed expression vector pcDNA3 as the backbone for the donor vector. Providing a detailed protocol for custom donor vector design and construction will enable researchers to develop unique genome editing tools. To date, detailed protocols for CRISPR Cas9 custom donor vector construction are limited (Lee et al. in Sci Rep 5:8572, 2015; Ma et al. in Sci Rep 4:4489, 2014). Custom donor vectors are commercially available, but can be expensive. Our goal is to share this protocol to aid researchers in performing genetic investigations that require custom donor vectors for specialized applications (specific gene truncations, knock-in mutations, and epitope tagging applications).

Keywords: CRISPR Cas9, Mammalian cell lines, Custom donor vector design and construction

\footnotetext{
*Correspondence: megan.keniry@utrgv.edu

${ }^{\dagger}$ Neftali Vazquez, Lilia Sanchez and Rebecca Marks contributed equally to this work

${ }^{1}$ Department of Biology, University of Texas-Rio Grande Valley, 1201 W.

University Dr., Edinburg, TX 78539, USA

Full list of author information is available at the end of the article
} 


\section{Background}

A significant proportion of bacteria and archaea (roughly 40 and 90\% respectively) employ [1,2] CRISPR Cas9 mechanisms as an adaptive immunological response against virus and plasmid foreign DNA [3-10]. Researchers have exploited the CRISPR Cas9 molecular machinery to target genes in numerous organisms such as yeast, flies, worms, and mammals leading to groundbreaking discoveries [11-14]. Although other approaches have been utilized for genome editing for decades, CRISPR Cas9 technology has reshaped genetic engineering by providing a quick and facile tool, greatly accelerating research $[13,14]$.

Endogenous CRISPR Cas9 (and related) systems serve as an acquired immunological response [3-5, 15-18]. Invading DNA (from plasmids and viruses) becomes incorporated into the CRISPR locus of the prokaryotic genome. CRISPR loci typically have noncontiguous direct repeats and spacers that contain the invading DNA sequences [19]. Transcription of the CRISPR locus produces a pre-crRNA (crRNA $=$ CRISPR RNA) that base pairs with a trans-activating-crRNA (tracrRNA, also encoded by CRISPR system), leading to processing and incorporation into a Cas9-containing complex [20-22]. Many prokaryotes harbor specific endonucleases such as Cas9 that contain two domains: RuvC-like [an endonuclease domain named for an Escherichia coli (E. Coli) protein involved in DNA repair] and $\mathrm{HNH}$ (an endonuclease domain named for characteristic histidine and asparagine residues) to cleave foreign DNA [15]. Hybridized crRNA/ tracrRNA serves as a guide for Cas9 to cleave foreign DNA in a sequence-specific manner. With heterologous CRISPR Cas9 systems such as those utilized in human cells, a chimeric guide RNA (gRNA) is employed to target specific sequences [23]. The gRNA contains a fusion between tracrRNA and crRNA that enables specific targeting of Cas9 to a gene of interest [24].

Employing CRISPR Cas9 technology as a gene editing tool is a recent development in the field of molecular biology $[14,24]$. This tool has already had a transformative impact on research, allowing for the quick identification of mutations in wide-ranging experimental settings [23]. However, it has become increasingly evident that utilization of CRISPR Cas9 can lead to off-target effects [19, 25, 26]. CRISPR Cas9 can tolerate base pair mismatches between the gRNA and target sequences [25, 27, 28]. CRISPR technology utilizes the host DNA repair machinery to resolve DNA lesions, leading to the isolation of mutations [29]. One issue that we encountered when trying to mutate genes in cancer cell lines, widely reported by others, was that mutation frequencies vary widely depending on the methodology employed, the locus being mutated and screening methods [17, 29]. Chiang et al. observed mutation efficiencies without selection by green fluorescent protein (GFP)-based cell sorting of $1-4 \%$ in HAP1 (near diploid chronic myelogenous leukemia) and 2-22\% in U2OS (human bone osteosarcoma epithelial) cell lines [28, 30-32]. In these settings with low mutation frequencies, methods that employ selection (such as neomycin resistance) may be necessary to obtain enough mutants for study in a cost effective manner. Here, we describe a detailed protocol to construct a custom donor vector (using the pcDNA3 vector as the backbone) in order to truncate the gene of interest. We describe a streamlined screening process to isolate and validate mutants in settings with low mutation frequencies.

\section{Methods}

This protocol can be employed to specifically truncate genes of interest using a neomycin resistance cassette (NPTII, enabling selection of mutants with G418) in mammalian cell lines. Figure 1 shows the basic steps to truncate the gene of interest. First, a custom donor vector needs to be designed and constructed. Next, CRISPR Cas9 mutagenesis needs to be performed. Last, mutants must be isolated and validated. This protocol addresses multiple barriers found with employing CRISPR Cas9 to mutate genes. First, we needed to design a custom donor vector in order to obtain truncation mutants for our research. Although we found many protocols for CRISPR mutagenesis, we found a lack of published protocols that described custom donor vector construction in detail and purchasing a custom donor vector can be expensive [1, 2]. Secondly, we sought to minimize off-target effects [25,

\section{Truncating FOXO3 gene using CRISPR}

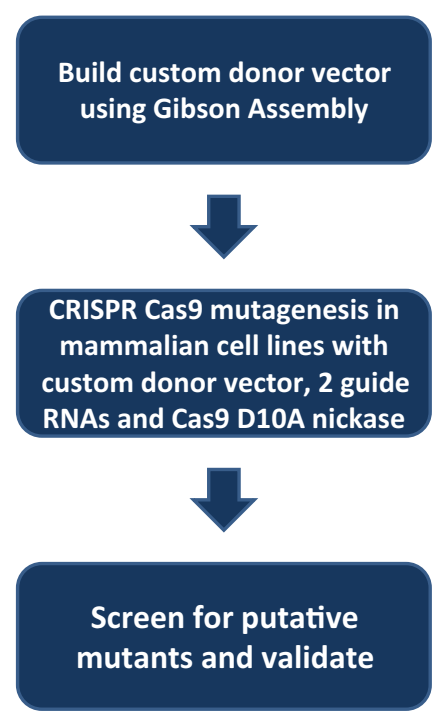

Fig. 1 Flow chart for CRISPR Cas9 mutagenesis using a custom donor vector. A flow chart for CRISPR mutagenesis with custom donor vector is depicted 
$28,30-32]$. Finally, we wanted to be able to quickly isolate mutants for a gene of interest, even if the mutation frequency was low. Many cancer cell lines such as U87MGs have deficient homologous recombination repair, making CRISPR mutagenesis inefficient [33, 34]. Our approach utilized transiently transfected Cas9D10A nickase, two gRNAs, and a donor vector to disrupt FOXO3 gene coding sequence with a neomycin resistance gene. The custom donor vector was built using two, separate Gibson assembly cloning steps with the pcDNA3 vector (Figs. 2, 3).

\section{Construction of $\mathrm{FOXO3}$ donor vector using Gibson assembly}

Gibson assembly is an extremely efficient method to obtain insertions into a plasmid vector of interest [35]. The FOXO3 donor vector was prepared using a two-step Gibson assembly-based cloning procedure. The complete sequence of the $\mathrm{FOXO3}$ donor vector can be found in Additional file 1: Figure S1. Figures 2, 3 depict the steps employed to prepare a custom $\mathrm{FOXO3}$ donor vector. First, a 418 base pair fragment from the FOXO3 gene (named FOXO3 Arm 1) was inserted into the pcDNA3 vector just upstream of the neomycin resistance cassette (NPTII), producing an intermediate vector. In the second sub-cloning step, (shown in Figs. 2, 3) a 750 base pair FOXO3 fragment (Arm 2) was inserted into the donor vector.

For optimal results, FOXO3 donor vector sequences were selected for (1) proximity to genomic nick sites and (2) sufficient sequence length to permit efficient recombination. It is important to note that there are two nick sites for $F O X O 3$ in the genome when using 2 gRNAs and the Cas9D10A nickase. Therefore, the upstream FOXO3 fragment (Arm 1) in the donor vector and downstream FOXO3 fragment (Arm 2) need to be in regions that are in positions amenable to recombination with the two genomic CRISPR nick sites. The FOXO3 donor vector fragments should be within 20 bases of the nick sites

\section{Donor Vector Construction using pcDNA3}

\section{Cut pcDNA3 vector with Dralll and purify fragment with spin column}

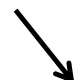

Amplify PCR fragment with flanking pcDNA3 sequences (near Dralll site) and purify with spin column
Cut vector with FOXO3 Arm 1 using BstZ17I and purify vector using spin column
Amplify PCR fragment with flanking pcDNA3 sequences (near BstZ17I site) and purify with spin column
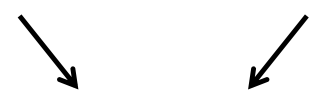

\section{Perform Gibson Assembly reaction to add second donor vector arm (Arm 2 of FOXO3) to obtain complete $\mathrm{FOXO} 3$ donor vector}

Fig. 2 Construction of $\mathrm{FOXO} 3$ donor vector. A fragment of FOXO3 (Arm 1) was inserted into the pCDNA3 vector proximal to the Drall restriction site by using Gibson assembly. This intermediate vector was called FOXO3 Arm1 and was utilized to make the final FOXO3 donor vector. Another fragment of FOXO3 (Arm 2) was inserted into the FOXO3 Arm1 vector (by Gibson assembly) to obtain the final FOXO3 donor vector. This vector was confirmed by Sanger sequencing and employed in CRISPR Cas9 mutagenesis reactions 


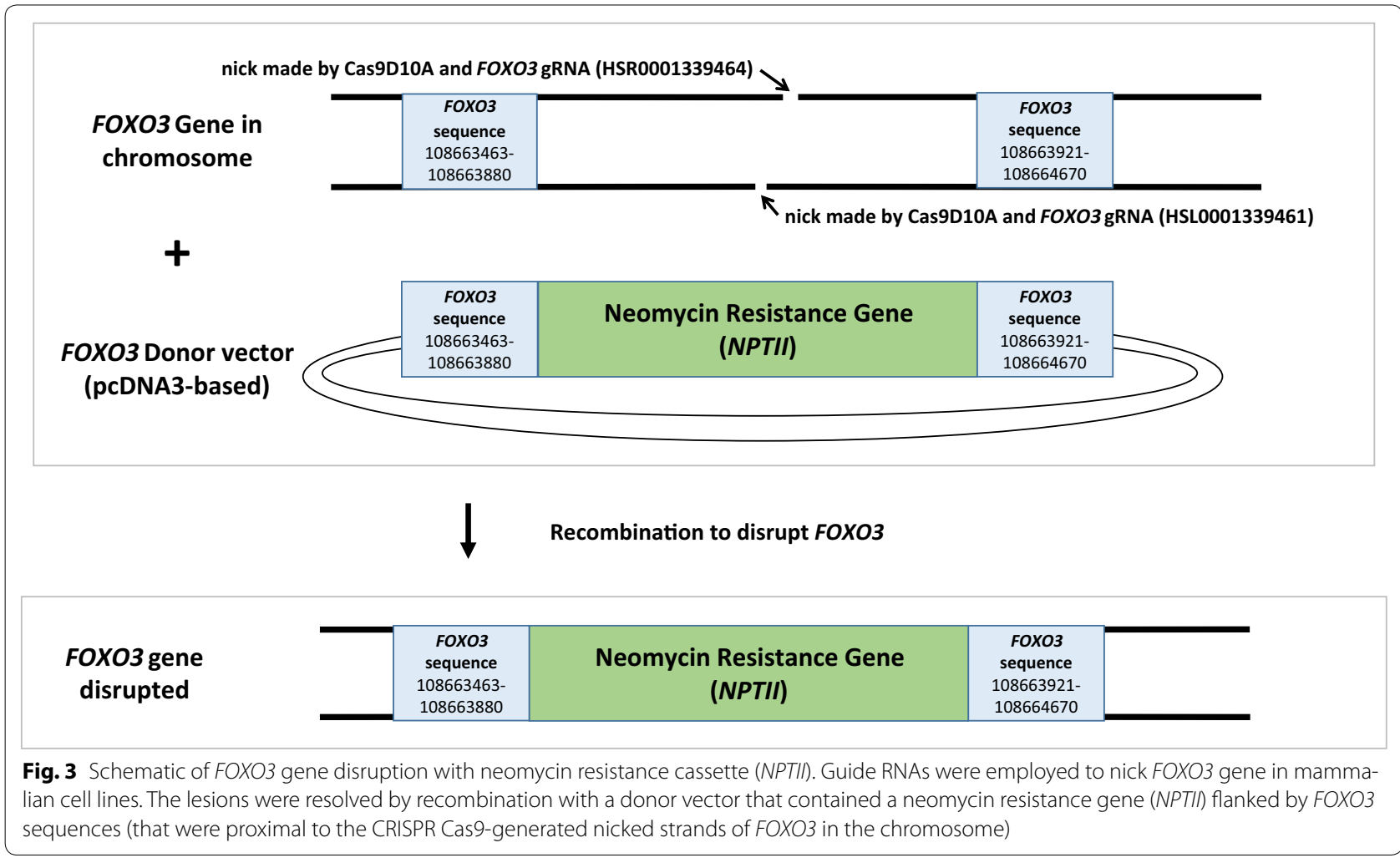

and should have at least a few hundred base pairs to promote recombination between the donor vector and the chromosome [26]. In our design, the upstream sequence used in the donor vector for FOXO3 Arm 1 contained 418 chromosomal FOXO3 base pairs; these sequences ended seven bases upstream of the gRNA targeting site in the genome, allowing for 418 base pairs of homology between the donor vector and genome for recombination-mediated repair just before the nick site in the genome. The distance between the bottom strand and the top strand nick sites made by CRISPR Cas9 D10A was 51 bases. The donor vector sequences in FOXO3 Arm 1 and FOXO3 Arm 2 were non-overlapping. The downstream fragment in the donor vector contained 750 bases that were homologous to FOXO3 chromosomal DNA (that extended beyond the second nick site further downstream into the gene) in order to promote recombination; the second nick site was 12 bases from the start of the FOXO3 Arm 2.

\section{Step-by-step Gibson assembly reactions for the FOXO3 donor vector}

Gibson assembly reactions were performed to insert two FOXO3 fragments into the pcDNA3 vector at positions that were on either side of neomycin resistance cassette (NPTII gene). For the Gibson assembly reaction, there needed to be identical sequences on the ends of each piece of DNA that would be physically joined. Therefore, the ends of each PCR product needed to be identical to the piece of pcDNA3 vector to which it would be fused. We added pcDNA3 vector sequences to the $5^{\prime}$ ends of PCR primers utilized to amplify FOXO3 fragments. Therefore, FOXO3 gene fragments (PCR products) had pcDNA3 sequences on the ends that corresponded to upstream and downstream sequences of the utilized restriction sites (DraIII for Arm1 and BstZ17I for Arm2) in pcDNA3.

\section{Addition of $\mathrm{FOXO} 3 \mathrm{Arm} 1$ to donor vector}

The pcDNA3 vector was cleaved with DraIII (restriction enzyme from NEB, Ipswich, MA) for $2 \mathrm{~h}$ at $37^{\circ} \mathrm{C}$. The restriction digest included $1 \mu \mathrm{g}$ of the pcDNA3 vector, $4 \mu \mathrm{L}$ of $10 \times$ NEB Cut Smart buffer, $3 \mu \mathrm{L}$ of DraIII restriction enzyme and $27 \mu \mathrm{L}$ of water. After this, $1 \mu \mathrm{L}$ of calf intestine phosphatase (CIP) was added to the reaction (from NEB, Ipswich, MA) and incubated for 1 more hour at $37^{\circ} \mathrm{C}$. The digested and phosphatased vector was column purified using Qiagen PCR purification system (Hilden, Germany). DNA was eluted with sterile water and quantified using a Nanodrop spectrophotometer.

In order to obtain the FOXO3 Arm 1 for sub-cloning, a PCR product was prepared that had a fragment of the FOXO3 gene (adjacent to the CRISPR nick sites in the 
genome) with sequences on the end of each primer that were identical to the pCDNA3 vector next to the DraIII site; see Table 1 for PCR primer sequences. The FOXO3 fragment was within 20 bases of the nick in the genome for recombination. This fragment was 418 base pairs in length, allowing a significant amount of homology to promote recombination between the donor vector and the nicked chromosome. The FOXO3 fragment was prepared using Phusion high fidelity polymerase (Thermo-Fisher, Waltham, MA). The FOXO3 Arm 1 PCR product was cleaved with $D p n \mathrm{I}$ for $3 \mathrm{~h}$ to remove plasmid DNA template (used as template for PCR reaction); $2 \mu \mathrm{L}$ of $D p n \mathrm{I}$ (NEB, Ipswich, MA) were added to unpurified FOXO3 PCR product (still containing nucleotides, polymerase, etc.) and samples were incubated at $37{ }^{\circ} \mathrm{C}$ for $2 \mathrm{~h}$. After $D p n I$ digestion, the PCR product was column purified using Qiagen PCR purification system (Hilden, Germany) and eluted with sterile water. The purified PCR product was quantified with a Nanodrop spectrophotometer.

Gibson assembly reactions were performed using $10 \mathrm{ng}$ of vector (cut, phosphatased and column purified pcDNA3) with $80 \mathrm{ng}$ of insert (FOXO3 fragment); the DNA reactants comprised a volume of $10 \mu \mathrm{L}$ initially. To the DNA reactants, $10 \mu \mathrm{L}$ of NEB Gibson assembly mix was added for a final volume of $20 \mu \mathrm{L}$ (NEB, Ipswich, MA). These reactions were incubated at $50{ }^{\circ} \mathrm{C}$ for $1 \mathrm{~h}$ and were then transformed into chemically competent bacterial cells (5-alpha competent E. coli, NEB, Ipswich, MA) as directed by the NEB Gibson assembly kit. Transformed bacterial cells were plated in dilutions $(1: 10,1: 100$ and 1:1000) to obtain single colonies given the high efficiency of the Gibson assembly reactions. Single colonies were screened by restriction digest and confirmed by Sanger sequencing. The vector obtained from this was called FOXO3 Arm 1 vector.

\section{Addition of $\mathrm{FOXO} 3 \mathrm{Arm} 2$ to donor vector}

The second arm for the FOXO3 donor vector was prepared in a similar manner to Arm 1. The intermediate

Table 1 PCR Primers utilized to amplify FOXO3 gene fragments for Donor Vector Gibson Assembly Reactions

\begin{tabular}{lc}
\hline Primer & Sequence $^{\mathbf{a}}$ \\
\hline FOXO3 ARM 1 F & 5'-GTGCTTTACGGCACCTCGACCCCCCGGCACAAC- \\
& CTGTCACTGC-3' \\
FOXO3 ARM 1 R & 5'-CCGTCTATCAGGGCGATGGCCGCTGTAGAGCAT \\
& GGGCGAGAG-3' \\
FOXO3 ARM 2 F & 5'-CAAATAAAGCATTTTTTTCACTCGGTGGAACTG \\
& CCACGGCTG-3' \\
FOXO3 ARM 2 R & 5'-GAGTTTGGACAAACCACAACTAGGTCCAAGT \\
& CGCTGGGAAC-3' \\
\hline
\end{tabular}

a Nucleotides in bold corresponds to pCDNA3 vector sequences. Sequences in italics were to amplify the indicated $\mathrm{FOXO} 3 \mathrm{arm}$ vector (with FOXO3 Arm 1) was cut with BstZ17I, which is on the other side of the neomycin resistance gene in the pcDNA3 plasmid compared to FOXO3 Arm 1. The cleaved vector was treated with CIP $(1 \mu \mathrm{L}$ CIP, NEB, Ipswich, MA) for $1 \mathrm{~h}$ and subsequently column purified. FOXO3 Arm 2 was amplified with the primer pair specified in Table 1, producing a product that had sequences on each end that were identical to the sequences proximal to the BstZ17I site in the intermediate FOXO3 Arm 1 vector. Gibson assembly reactions were performed (as previously described to sub-clone Arm 1 of FOXO3) to obtain the final FOXO3 donor vector Figs. 2, 3. Transformed bacterial cells were plated in dilutions $(1: 10,1: 100$ and 1:1000) to obtain single colonies given the extremely high efficiency of the Gibson assembly reactions. The complete $\mathrm{FOXO} 3$ donor vector was confirmed by restriction fragment analysis and Sanger sequencing. The complete sequence of the FOXO3 donor vector can be found in Additional file 1: Figure S1.

\section{CRISPR Cas9 mutagenesis to truncate the $\mathrm{FOXO3}$ gene in mammalian cells}

Transient transfections to obtain $\mathrm{FOXO}$ truncation mutants

Transient transfections were performed using the glioblastoma cell line U87MG, breast cancer cell line BT549, or human kidney cell line HEK 293, which were obtained from American Type Culture Collection (ATCC, Manassas, VA) and propagated under standard conditions [ $37{ }^{\circ} \mathrm{C}$ with $5 \% \mathrm{CO}_{2}$ in media specified by ATCC supplemented with 10\% FBS (fetal bovine serum) and 5\% penicillin/streptomycin (pen/strep)]. Media employed to grow U87MG cells was MEM, BT549 was RPMI and HEK 293 was DMEM. One million cells of each cell line was transfected using LONZA (nucleofection kit V, program P-20 for U87MG and BT549 and program X-001 for HEK 293) and were allowed to recover for 2 days in $10 \mathrm{~cm}$ dishes. In each transfection, $250 \mathrm{ng}$ of each guide RNA (gRNA) vector (Table 2) were added as well as $250 \mathrm{ng}$ of the vector enabling Cas9D10A expression (CRISPR Cas9D10A-GFP Nickase, catalog: CAS9D10AGFPP, Sigma, St. Louis, MO). Guide RNA vectors were obtained from Sigma (St. Louis, MO) and were utilized to make DNA nicks in both chromosomal FOXO3 DNA strands (Figs. 2, 3). The FOXO3-containing portions of the gRNAs are detailed in Table 2. A negative control gRNA (CRISPR Universal Negative Control 1, catalog: CRISPR06, Sigma, St. Louis MO) was utilized for control experiments (500 ng per transfection to have the same amount of DNA as mutagenesis samples). One microgram of $\mathrm{FOXO} 3$ donor vector was utilized in each transfection. 
Table 2 Guide RNA sequences

\begin{tabular}{lll}
\hline Gene & I.D. for construct & Sequence \\
\hline FOXO3 & HSL0001339461 & CTTACTGAAGGTGACAGGCTGG \\
FOXO3 & HSR0001339464 & CACGGCTGACTGATATGGCAGG \\
\hline
\end{tabular}

It is important to note that the nicks directed by the gRNAs are staggered on the chromosome (about 40 base pairs apart). These gRNAs are used in concert with the Cas9D10A nickase (CRISPR Cas9D10A-GFP Nickase, catalog: CAS9D10AGFPP, Sigma, St. Louis, MO) to make nicks in a gene of interest [26, 28, 30-32]. It has been shown that Cas9D10A allows for $>100$-fold increased specificity for genomic editing (between 200-fold and 1500-fold based on deep sequencing experiments) [26].

For each mutagenesis, cells that survived the transfection (after 2 days of recovery) were incubated in $0.25 \%$ trypsin for $3 \mathrm{~min}$ and were placed into $10 \mathrm{~mL}$ of MEM (contained 10\% FBS and 5\% Pen/Strep). Ten plates of diluted cells (approximately 100,000 cells per $10 \mathrm{~cm}$ dish) were prepared from this mixture. G418 (0.5 mg/ $\mathrm{mL}$ final concentration for U87MG and BT549 and $1.5 \mathrm{mg} / \mathrm{mL}$ G418 for HEK 293 cells) was added to each $10 \mathrm{~cm}$ plate 1 day after plating. Single clones were isolated from these selected dishes 4 weeks later using cloning cylinders. 2-10 single colonies were obtained from each $10 \mathrm{~cm}$ plate. To clone a colony, the plate was washed with $2 \mathrm{~mL} 0.25 \%$ trypsin and aspirated. Cloning cylinders (Fisher: 0955221) were placed onto the $10 \mathrm{~cm}$ plate using sterile forceps and vaseline (to make the cylinder stick to the plate). $200 \mu \mathrm{L}$ of $0.25 \%$ trypsin was added to each cylinder and incubated for $5 \mathrm{~min}$. The $200 \mu \mathrm{L}$ of trypsin was pipetted up and down ten times and then plated into $2 \mathrm{~mL}$ of fresh media in a well of a six well plate.

\section{Results}

\section{Western blot analysis with putative FOXO3 mutants}

Proof-of-principle studies were done to determine mutation frequencies using our described protocol in several mammalian cell lines: U87MG, BT549 and HEK 293. Figure 4 depicts the encoded FOXO3 truncation mutant protein. FOXO3 is an extensively characterized transcriptional activator that impacts metabolism, the cell cycle, apoptosis and cell fate [36-38]. Disruption of the FOXO3 gene led to the production of a truncated mutant protein that contained the first 349 amino acids of FOXO3 (including the DNA binding domain); most of the transactivation domain was deleted. CRISPR Cas9 mutagenesis followed by selection with neomycin with U87MG cells allowed our group to isolate 77 putative mutants in one trial and 50 putative mutants in another. We confirmed the truncation mutants in several ways. Western blot analyses were performed as described previously [39]. Expression of FOXO3 was assessed by western blot analysis. Total protein was obtained from U87MG cells by rinsing out the 6 well plate wells with 1XPBS followed by directed lysis in $2 \times$ sample buffer $(125 \mathrm{mM}$ Tris-HCL at $\mathrm{pH} 6.8,2 \%$ sodium dodecyl sulfate (SDS), $10 \%$ 2-mercaptoethanol, 20\% glycerol, 0.05\% bromophenol blue, $8 \mathrm{M}$ urea); $2 \times$ sample buffer was added to each well and scraped. The lysate was collected from each well, placed into $1.5 \mathrm{~mL}$ microcentrifuge tubes and heated for 10 min at $90{ }^{\circ} \mathrm{C}$ in a dry-bath heat block. Equal amounts of protein lysates were separated by sodium dodecyl sulfate-polyacrylamide gel (SDS-PAGE) electrophoresis at $100 \mathrm{~V}$ for $1 \mathrm{~h}$. The protein was then transferred onto a polyvinylidene fluoride (PVDF) membrane for an hour and 30 min then blocked in a 5\% milk solution [carnation powdered milk, $1 \times$ tri-buffered saline with Tween 20 (TBST)] for an hour. The membrane was incubated with FOXO3 (Cell Signaling, Danvers, MA, Cat: 75D8), or GAPDH (Santa Cruz Biotechnology, Dallas, TX, cat: G-9) antibodies overnight at $4{ }^{\circ} \mathrm{C}$ then washed for $20 \mathrm{~min}$ with TBST in 5-min intervals. The blot was incubated with secondary antibodies. The membrane was washed again for $20 \mathrm{~min}$ in 5-min intervals and allowed to develop using SuperSignal West Dura Extended Duration Substrate luminol solution for $5 \mathrm{~min}$. The BioRad ChemDoc XRS+ molecular imager was used to detect light emitted from protein-containing complexes. Western blot SCN files from the (BioRad ChemDoc XRS+) were analyzed using NIH Image J.

Western blot analysis showed that FOXO3 truncation mutant protein was much smaller than the wildtype FOXO3, roughly $45 \mathrm{kDa}$ (kilo Dalton) versus the full-length $80 \mathrm{kDa}$, Fig. 4. In our first attempt to isolate FOXO3 disruption mutants, we screened 77 putative clones and obtained four homozygous mutants based on western blot analysis, Fig. 4 . Therefore, $5 \%$ of the screened isolates were homozygous mutant. In a repeat proof-ofprinciple experiment, we found that out of 50 screened putative mutants, three had only the truncated form of FOXO3 as evidenced by the $45 \mathrm{kDa}$ band (6\% homozygous mutation rate), Fig. 5 . Three samples had both the truncated form of FOXO3 and the full-length FOXO3 protein (two bands that were 45 and $80 \mathrm{kDa}$ ), Fig. 5. 44 out of the 50 screened samples had full-length FOXO3 protein (only $80 \mathrm{kDa}$ band), Fig. 5. Therefore, even with selection, there was a low mutation rate $(5-6 \%)$. This was not surprising given the DNA repair deficiency found in U87MG cells [33, 34]. We also screened for FOXO3 truncation mutants in two additional cell lines: breast cancer BT549 and human kidney HEK 293. Out of 56 screened isolates in BT549 cells, five were homozygous mutant (approximately 9\%), Table 3 . We found that only one of 
a

Wild-type FOXO3 protein (673 a.a.)

$\downarrow$ CRISPR mutagenesis

Truncated FOXO3 protein (349 a.a.)

b

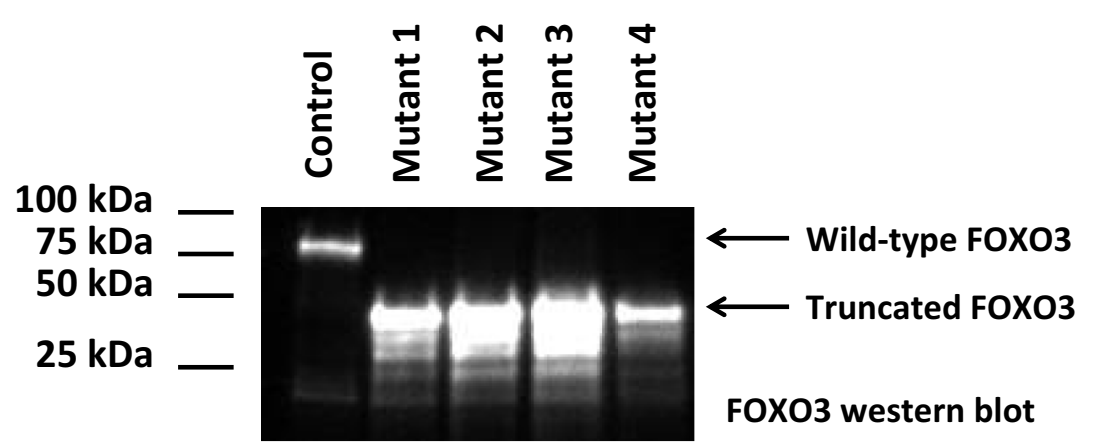

GAPDH western blot

Fig. 4 FOXO3 truncation mutant proteins retain the DNA binding domain. a Disruption of the FOXO3 gene as described in "Methods" led to a truncated protein that was 349 amino acids in length. This mutant protein retained the FOXO3 DNA binding domain, but lacked the transactivation domain. b Total protein lysates prepared from FOXO3 mutant-containing cells and control cells were examined by western blot analysis; employed antibodies are indicated. Wild-type FOXO3 was approximately $80 \mathrm{kDa}$, whereas, mutant FOXO3 protein was approximately $45 \mathrm{kDa}$. Out of 77 putative mutants screened, only four were homozygous mutant (confirmed by Sanger sequencing)

77 putative mutants screened in HEK 293 background was homozygous for FOXO3 truncation, Table 3. Therefore the mutant frequency was between 1.3 and $9 \%$ in the tested cell lines.

In our experiments, we were able to screen for a change in protein size. Other applications of this protocol could use similar screening techniques when the mutation frequency is low. Attachment of a GFP fusion to a gene of interest could be screened by western blot analysis, microscopy or flow cytometric analysis. Alternatively, genes could be deleted, leading to a loss of a protein of interest in western blot analysis. The ability to select mutants with neomycin and then screen using western blot analysis (or other technique) greatly facilitates the isolation of mutants.

\section{Genotyping CRISPR Cas9 mutants}

All putative homozygous FOXO3 disruption mutants that were identified by western blot analyses (Figs. 4, 5 , and Table 3) were further confirmed using PCR and were later confirmed by Sanger sequencing. DNA was isolated from 1 million cells from each clone. The cells were removed from plates using trypsin and centrifuged at $700 \times g$ for $5 \mathrm{~min}$. Cells were re-suspended in $500 \mu \mathrm{L}$ of Buffer (10 mM Tris 7.4, $10 \mathrm{mM} \mathrm{NaCl}, 25 \mathrm{mM}$ EDTA, 1\% SDS). $25 \mu \mathrm{L}$ of Proteinase K (50 U/mL stock, catalog: 03115828001, Roche, Mannheim, Germany) was added to each sample followed by an $18 \mathrm{~h}$ incubation at $37^{\circ} \mathrm{C}$. Next, the sample was extracted with an equal volume of phenol, followed by centrifugation at top speed in phase lock tubes (Quanta Biosciences, Beverley, MA, catalog: 2302820). DNA was precipitated from the supernatant by adding $3 \mu \mathrm{L}$ of $20 \mathrm{mg} / \mathrm{mL}$ glycogen, $50 \mu \mathrm{L}$ of sodium acetate and $1 \mathrm{~mL}$ of $100 \%$ ethanol (stored at $-80{ }^{\circ} \mathrm{C}$ overnight followed by $20 \mathrm{~min}$ of centrifugation at $17,000 \times g$ ). The DNA pellets were washed with $70 \%$ ethanol, dried and re-suspended in sterile water. PCR was performed to identify clones that had NPTII integration into the FOXO3 locus using the primers in Table 4. The forward primer for PCR was approximately sixty bases upstream of the FOXO3 fragment found in Arm 1 of the FOXO3 donor vector. Therefore, the primer sequences employed 


\section{Screening for $\mathrm{FOXO3} \mathrm{disruption} \mathrm{mutants} \mathrm{in} \mathrm{U87MG} \mathrm{cells}$}
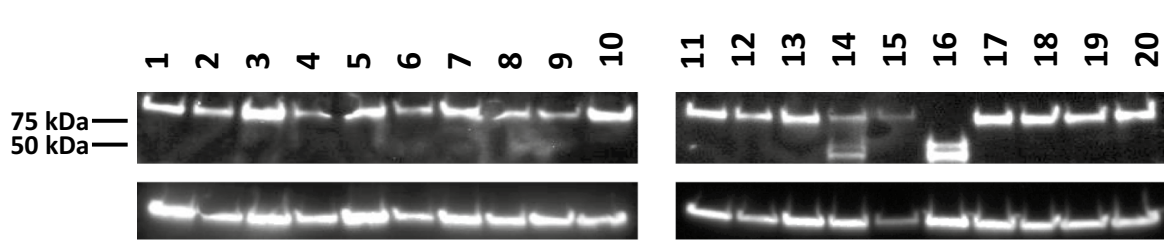

Antibody

FOXO3

GAPDH

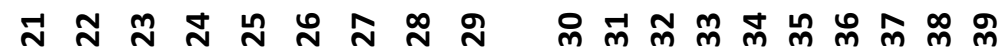
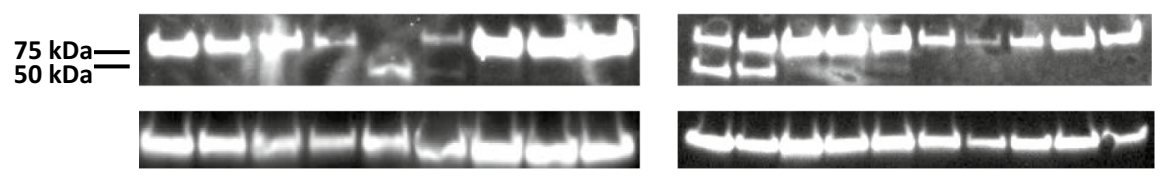

FOXO3

GAPDH
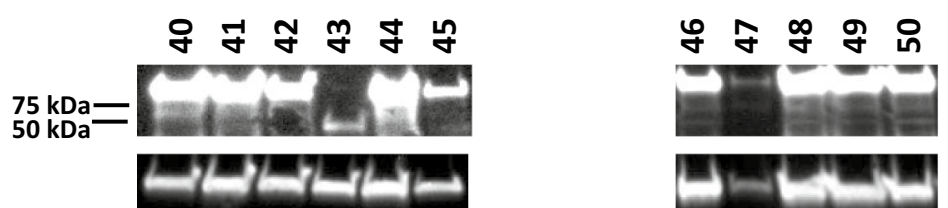

FOXO3

GAPDH

Fig. 5 Western blot screening for putative FOXO3 truncation mutants. Total protein lysates were prepared from 50 putative FOXO3 mutant-containing cells and were examined by western blot analyses; employed antibodies are indicated. Wild-type FOXO3 was approximately $80 \mathrm{kDa}$, whereas, mutant FOXO3 protein was approximately $45 \mathrm{kDa}$. This second proof of principle screen examined 50 potential FOXO3 mutant clones. Three of these were homozygous mutant (16, 25 and 43), three appeared to be heterozygous (14, 30 and 31) and 44 were wild-type. The homozygous mutants were confirmed by Sanger sequencing

Table 3 CRISPR Cas9 mutation frequencies in mammalian cell lines

\begin{tabular}{llll}
\hline Cell line & $\begin{array}{l}\text { G418-resistant } \\
\text { isolates screened } \\
\text { by western blot } \\
\text { analysis }\end{array}$ & $\begin{array}{l}\text { Number } \\
\text { of homozygous } \\
\text { truncation } \\
\text { mutants }\end{array}$ & $\begin{array}{l}\text { Homozygous } \\
\text { mutation } \\
\text { frequency } \\
\text { (\%) }\end{array}$ \\
\hline U87MG Trial 1 & 77 & $4^{\mathrm{a}}$ & 5 \\
U87MG Trial 2 & 50 & $3^{\mathrm{a}}$ & 6 \\
BT549 & 56 & 5 & 9 \\
HEK293 & 77 & 1 & 1.3 \\
\hline
\end{tabular}

a These mutants are shown in western blot analyses found in Figs. 4, 5

Table 4 Primers utilized to detect and sequence FOXO3 gene disruption

\begin{tabular}{ll}
\hline Primer name & Sequence \\
\hline FOXO3 F (for detection of disruption) & $5^{\prime}$-GTGCTTCAGGATCGCTTCA-3' \\
$\begin{array}{l}\text { Neo cassette R (for detection of } \\
\text { disruption) }\end{array}$ & $5^{\prime}$-TGCATGCTTTGCATACTTCTG-3' \\
$\begin{array}{l}\text { FOXO3 seq. (for sequencing disruption } \\
\text { mutants) }\end{array}$ & 5'-CTCGGTTTTGGACCATTCTG-3' \\
\hline
\end{tabular}

for mutant detection by PCR were absent from the donor vector and were only found in chromosomal FOXO3. The reverse primer was part of the NPTII cassette used to disrupt FOXO3. Most of the negative samples were positive for intact vector integration (data not shown). The PCR products (employed for detecting $\mathrm{FOXO} 3$ disruption) were column purified using Qiagen PCR purification system (Hilden, Germany) and eluted with sterile water. The purified PCR product was quantified with a Nanodrop spectrophotometer. Purified PCR products were analyzed by Sanger sequencing; a primer containing FOXO3 chromosomal sequences (that were not present in the donor vector) was utilized, Table 4. Sanger sequencing confirmed that the homozygous FOXO3 mutants identified by western blot analyses had the designed gene disruptions.

\section{Discussion}

CRISPR Cas9 technology is an emergent genome editing tool. Here, we describe a protocol to disrupt the FOXO3 gene in mammalian cells using a neomycin cassette. To decrease off-target effects, we employed 2 guide RNAs, a mutant Cas9D10A nickase and a FOXO3 donor vector that was constructed by Gibson assembly (to enable 
the selection of mutants with G418). Selected mutants were validated by PCR, Sanger sequencing and western blot analysis. This protocol could be adapted to readily disrupt or modify genes of interest in order to alter the genetic background of mammalian cell lines in a directed manner. The ability to select for the disruption of genes using neomycin resistance accelerates mutant isolation, especially when mutation frequencies are low or when mutations are deleterious to cells.

Many cancer cell lines including U87MGs have deficient DNA repair [33, 34]. Homozygous mutation frequencies varied depending on the cell line as seen in Table 3. We found that even with selection, $5-6 \%$ of screened putative mutants were homozygous for $\mathrm{FOXO} 3$ disruption in U87MG cells (Figs. 4, 5, Table 3). BT549 breast cancer cells had the highest efficiency of $9 \%$. HEK 293 cells had the lowest homozygous mutation frequency of only $1.3 \%$ (Table 3 ), whereas many heterozygous mutants were obtained for this cell line ( 8 out of 77 screened by western blot, data not shown). Similar homology directed repair (HDR) frequencies were observed in HEK 293 backgrounds (0.2-1.5\%) with the Cas9 D10A nickase in previous studies [24, 40, 41]. HDR frequencies using CRISPR Cas9 vary depending on the cell line, enzymes utilized (Cas9 D10A versus wild-type Cas9), transfection protocols employed, specific guide RNAs employed (including PAM sequence variances) and the specific locus being mutated [23, 27, 29, 40]. It was surprising that U87MG and BT549 cell lines had higher gene disruption frequencies than HEK 293 cells. Perhaps NHEJ more efficiently resolved the Cas9-derived nicks in HEK 293 cells, leading to lower homology directed repair in this setting. NHEJ frequencies were found to be higher than HDR frequencies in 293 backgrounds (50-60\% compared to $1 \%$, respectively) [40]. In addition, BT549 and U87MG cells harbor null mutations in the tumor suppressor PTEN, which impacts DNA repair via numerous mechanisms in a context-dependent manner [33, 43-46]. Loss of PTEN hinders DNA repair, which may shift double strand break resolution to favor HDR over NHEJ in U87MG and BT549 cell lines [33].

\section{Conclusions}

We describe a CRISPR Cas9 genome editing protocol for mammalian cell lines by constructing and employing a custom donor vector that contains a neomycin resistance cassette. We provide a detailed, step-by-step protocol for donor vector design and construction using the pcDNA3 vector [47]. Custom donor vectors can be difficult to clone and expensive to purchase. We provide a simple, efficient protocol to obtain custom donor vectors from the common pcDNA3 mammalian expression vector. We also provide step-by-step instructions on how to select mutants and isolate clones. This protocol will allow researchers to overcome the barrier of low mutation efficiency commonly found in mammalian cell lines. Importantly, researchers can employ this protocol to build custom donor vectors in order to study novel gene functions and/or examine the localization of tagged proteins using endogenous expression levels.

\section{Additional file}

Additional file 1: Figure S1. FOXO3 donor vector complete sequence. The complete sequence of $\mathrm{FOXO} 3$ donor vector is provided. FOXO3 Arm 1 and Arm 2 are indicated. NPTII (neomycin resistance cassette) is indicated.

\section{Abbreviations}

CRISPR: clustered regularly interspaced short palindromic repeats; Cas9: CRISPR associated sequence 9; NHEJ: non homologous end joining; HR: homologous recombination DNA repair; NPTII: Neomycin Phosphotransferase II; FOXO3: forkhead box O3; G418: geneticin; PCR: polymerase chain reaction; U87MG: Uppsala 87 Malignant Glioma; crRNA: CRISPR RNA; tracrRNA: trans activating CRISPR RNA; gRNA: guide RNA (for heterologous systems); RuvC: an endonuclease domain named for an E. coli protein involved in DNA repair; $\mathrm{HNH}$ : an endonuclease domain named for characteristic histidine and asparagine residues; GFP: green fluorescent protein; HAP1: near-haploid human cell line; U2OS: human bone osteosarcoma epithelial cells; CIP: calf intestine phosphatase; E. coli: Escherichia coli; ATCC: American Type Culture Collection; MEM: minimal essential media; FBS: fetal bovine serum; Pen/strep: penicillin/ streptomycin; kDa: kilo Dalton; IBC: Institutional Biosafety Committee.

\section{Authors' contributions}

$M K, R G, W I, E S$, and RD formulated the hypothesis, organized the study, designed the protocol, analyzed the data and wrote the manuscript. NV, RM, $E M, V F, L S, A L, A S$, IF, and JH performed the experiments. All authors read and approved the final manuscript.

\section{Author details \\ ${ }^{1}$ Department of Biology, University of Texas-Rio Grande Valley, 1201 W. University Dr., Edinburg, TX 78539, USA. ${ }^{2}$ Department of Biology, Montclair State University, 1 Normal Ave., Montclair, NJ 07043, USA. ${ }^{3}$ School of Medicine, University of Texas-Rio Grande Valley, 1201 W. University Dr., Edinburg, TX 78539, USA.}

\section{Acknowledgements}

The authors would like to thank the UTRGV Department of Biology and COS for their support, reagents and expertise.

Competing interests

The authors declare that they have no competing interests.

Availability of data and materials

All cell lines and additional data prepared from this work are available upon request.

Consent for publication

Not applicable.

\section{Ethics approval and consent to participate}

Work was performed with Institutional Biosafety Committee approval from the University of Texas Rio Grande Valley: Registration Number: 2016-003-IBC.

\section{Funding}

This work was supported by HHMI 52007568 (N.V. and R.M.), USDA Step 2 2015-38422-24061 (A.L., R.G.), USDA H.S.I. 2016-38422-25760 (M.K. and E.M), NIH 5R25GM10086606 (A.S., I.F and R.D.), NIH 5SC3GM11666901 (R.G.), UTRGV College of Sciences (COS) Seed Grant (M.K.), NSF Advance 1209210 (M.K.), and NSF 1463991 (E.S and M.K.). 


\section{Publisher's Note}

Springer Nature remains neutral with regard to jurisdictional claims in published maps and institutional affiliations.

Received: 16 August 2017 Accepted: 6 March 2018

Published online: 14 March 2018

\section{References}

1. Lee JS, et al. Site-specific integration in CHO cells mediated by CRISPR/ Cas9 and homology-directed DNA repair pathway. Sci Rep. 2015;5:8572. https://doi.org/10.1038/srep08572.

2. Ma S, et al. CRISPR/Cas9 mediated multiplex genome editing and heritable mutagenesis of BmKu70 in Bombyx mori. Sci Rep. 2014;4:4489. https://doi.org/10.1038/srep04489.

3. Jansen $\mathrm{R}$, et al. Identification of genes that are associated with DNA repeats in prokaryotes. Mol Microbiol. 2002;43(6):1565-75.

4. Sorek R, Kunin V, Hugenholtz P. CRISPR-a widespread system that provides acquired resistance against phages in bacteria and archaea. Nat Rev Microbiol. 2008;6(3):181-6. https://doi.org/10.1038/nrmicro1793.

5. van der Oost J, et al. CRISPR-based adaptive and heritable immunity in prokaryotes. Trends Biochem Sci. 2009;34(8):401-7. https://doi. org/10.1016/j.tibs.2009.05.002

6. Mojica FJ, Diez-Villasenor C. The on-off switch of CRISPR immunity against phages in Escherichia coli. Mol Microbiol. 2010;77(6):1341-5.

7. Mojica FJ, et al. Short motif sequences determine the targets of the prokaryotic CRISPR defence system. Microbiology. 2009;155(Pt 3):733-40. https://doi.org/10.1099/mic.0.023960-0.

8. Mojica FJ, et al. Intervening sequences of regularly spaced prokaryotic repeats derive from foreign genetic elements. J Mol Evol. 2005;60(2):17482. https://doi.org/10.1007/s00239-004-0046-3.

9. Mojica FJ, Montoliu L. On the origin of CRISPR-Cas technology: from prokaryotes to mammals. Trends Microbiol. 2016;24(10):811-20. https:// doi.org/10.1016/j.tim.2016.06.005.

10. Mojica FJ, Rodriguez-Valera F. The discovery of CRISPR in archaea and bacteria. FEBS J. 2016;283(17):3162-9. https://doi.org/10.1111/febs.13766.

11. Frokjaer-Jensen C. Exciting prospects for precise engineering of Caenorhabditis elegans genomes with CRISPR/Cas9. Genetics. 2013;195(3):63542. https://doi.org/10.1534/genetics.113.156521.

12. Sternberg SH, Doudna JA. Expanding the biologist's toolkit with CRISPR-Cas9. Mol Cell. 2015;58(4):568-74. https://doi.org/10.1016/j. molcel.2015.02.032.

13. Wright AV, Nunez JK, Doudna JA. Biology and applications of CRISPR systems: harnessing nature's toolbox for genome engineering. Cell. 2016;164(1-2):29-44. https://doi.org/10.1016/j.cell.2015.12.035.

14. Qi LS, et al. Repurposing CRISPR as an RNA-guided platform for sequence-specific control of gene expression. Cell. 2013;152(5):1173-83. https://doi.org/10.1016/j.cell.2013.02.022.

15. Horvath P, Barrangou R. CRISPR/Cas, the immune system of bacteria and archaea. Science. 2010;327(5962):167-70. https://doi.org/10.1126/ science.1179555.

16. Bhaya D, Davison M, Barrangou R. CRISPR-Cas systems in bacteria and archaea: versatile small RNAs for adaptive defense and regulation. Annu Rev Genet. 2011;45:273-97. https://doi.org/10.1146/ annurev-genet-110410-132430.

17. Mali $P$, et al. RNA-guided human genome engineering via Cas9. Science. 2013;339(6121):823-6. https://doi.org/10.1126/science.1232033.

18. Sinkunas T, et al. In vitro reconstitution of Cascade-mediated CRISPR immunity in Streptococcus thermophilus. EMBO J. 2013;32(3):385-94. https://doi.org/10.1038/emboj.2012.352.

19. FuY, et al. High-frequency off-target mutagenesis induced by CRISPR-Cas nucleases in human cells. Nat Biotechnol. 2013;31(9):822-6. https://doi. org/10.1038/nbt.2623

20. Koo T, Lee J, Kim JS. Measuring and reducing off-target activities of programmable nucleases including CRISPR-Cas9. Mol Cells. 2015;38(6):47581. https://doi.org/10.14348/molcells.2015.0103.

21. Deltcheva E, et al. CRISPR RNA maturation by trans-encoded small RNA and host factor RNase III. Nature. 2011;471(7340):602-7. https://doi. org/10.1038/nature09886.
22. Touchon M, et al. CRISPR distribution within the Escherichia coli species is not suggestive of immunity-associated diversifying selection. J Bacteriol. 2011;193(10):2460-7. https://doi.org/10.1128/JB.01307-10.

23. Cong L, Zhang F. Genome engineering using CRISPR-Cas 9 system. Methods Mol Biol. 2015;1239:197-217. https://doi. org/10.1007/978-1-4939-1862-1_10.

24. Ran FA, et al. Genome engineering using the CRISPR-Cas9 system. Nat Protoc. 2013;8(11):2281-308. https://doi.org/10.1038/nprot.2013.143.

25. Peng R, Lin G, Li J. Potential pitfalls of CRISPR/Cas9-mediated genome editing. FEBS J. 2016;283(7):1218-31. https://doi.org/10.1111/febs.13586.

26. Ran FA, et al. Double nicking by RNA-guided CRISPR Cas9 for enhanced genome editing specificity. Cell. 2013;154(6):1380-9. https://doi. org/10.1016/j.cell.2013.08.021.

27. Zhang XH, et al. Off-target effects in CRISPR/Cas9-mediated genome engineering. Mol Ther Nucleic Acids. 2015;4:e264. https://doi. org/10.1038/mtna.2015.37.

28. Chiang TW, et al. CRISPR-Cas 9(D10A) nickase-based genotypic and phenotypic screening to enhance genome editing. Sci Rep. 2016;6:24356 https://doi.org/10.1038/srep24356.

29. Cong $L$, et al. Multiplex genome engineering using CRISPR/Cas systems. Science. 2013;339(6121):819-23. https://doi.org/10.1126/ science. 1231143.

30. Nagarajan S, et al. BRD4 promotes p63 and GRHL3 expression downstream of FOXO in mammary epithelial cells. Nucleic Acids Res. 2017;45(6):3130-45. https://doi.org/10.1093/nar/gkw1276.

31. Wu CW, et al. Corrigendum to "Inactivation of p53 in pterygium influence miR-200a expression resulting in ZEB1, ZEB2 up-regulation and EMT processing" [Exp. Eye Res. 146 (2016) 206-211]. Exp Eye Res. 2016;151:256. https://doi.org/10.1016/j.exer.2016.06.005.

32. Wu CW, et al. Inactivation of p53 in pterygium influence miR-200a expression resulting in ZEB1/ZEB2 up-regulation and EMT processing. Exp Eye Res. 2016;146:206-11. https://doi.org/10.1016/j.exer.2016.03.012.

33. Bassi $C$, et al. Nuclear PTEN controls DNA repair and sensitivity to genotoxic stress. Science. 2013;341(6144):395-9. https://doi.org/10.1126/ science.1236188.

34. Majuelos-Melguizo J, et al. PARP targeting counteracts gliomagenesis through induction of mitotic catastrophe and aggravation of deficiency in homologous recombination in PTEN-mutant glioma. Oncotarget. 2015;6(7):4790-803. https://doi.org/10.18632/oncotarget.2993.

35. Grozdanov PN, MacDonald CC. Generation of plasmid vectors expressing FLAG-tagged proteins under the regulation of human elongation factor1 alpha promoter using Gibson assembly. J Vis Exp. 2015. https://doi. org/10.3791/52235

36. Calnan DR, Brunet A. The FoxO code. Oncogene. 2008:27(16):2276-88. https://doi.org/10.1038/onc.2008.21

37. Calnan DR, et al. Methylation by Set9 modulates FoxO3 stability and transcriptional activity. Aging (Albany NY). 2012;4(7):462-79. https://doi. org/10.18632/aging.100471

38. Carter ME, Brunet A. FOXO transcription factors. Curr Biol. 2007;17(4):R113-4. https://doi.org/10.1016/j.cub.2007.01.008

39. Keniry M, et al. Survival factor NFIL3 restricts FOXO-induced gene expression in cancer. Genes Dev. 2013;27(8):916-27. https://doi.org/10.1101/ gad.214049.113.

40. Zaboikin M, et al. Non-homologous end joining and homology directed DNA repair frequency of double-stranded breaks introduced by genome editing reagents. PLoS ONE. 2017;12(1):e0169931. https://doi. org/10.1371/journal.pone.0169931.

41. Miyaoka Y, et al. Systematic quantification of HDR and NHEJ reveals effects of locus, nuclease, and cell type on genome-editing. Sci Rep. 2016:6:23549. https://doi.org/10.1038/srep23549.

42. Li J, et al. PTEN, a putative protein tyrosine phosphatase gene mutated in human brain, breast, and prostate cancer. Science. 1997;275(5308):1943-7

43. Saal LH, et al. Recurrent gross mutations of the PTEN tumor suppressor gene in breast cancers with deficient DSB repair. Nat Genet. 2008:40(1):102-7. https://doi.org/10.1038/ng.2007.39.

44. Saal LH, et al. PIK3CA mutations correlate with hormone receptors, node metastasis, and ERBB2, and are mutually exclusive with PTEN loss in human breast carcinoma. Cancer Res. 2005;65(7):2554-9. https://doi. org/10.1158/0008-5472-CAN-04-3913. 
45. Kang YJ, et al. Contribution of classical end-joining to PTEN inactivation in p53-mediated glioblastoma formation and drug-resistant survival. Nat Commun. 2017;8:14013. https://doi.org/10.1038/ncomms14013.

46. Shen WH, et al. Essential role for nuclear PTEN in maintaining chromosomal integrity. Cell. 2007;128(1):157-70. https://doi.org/10.1016/j. cell.2006.11.042.
47. Goldman LA, et al. Modifications of vectors pEF-BOS, pcDNA1 and pcDNA3 result in improved convenience and expression. Biotechniques. 1996;21(6):1013-5.

\section{Submit your next manuscript to BioMed Central and we will help you at every step:}

- We accept pre-submission inquiries

- Our selector tool helps you to find the most relevant journal

- We provide round the clock customer support

- Convenient online submission

- Thorough peer review

- Inclusion in PubMed and all major indexing services

- Maximum visibility for your research

Submit your manuscript at

www.biomedcentral.com/submit 